\title{
A Mobile DNP Polarizer for Clinical Applications
}

\author{
K. Münnemann ${ }^{1}$, C. Bauer², J. Schmiedeskamp², H. W. Spiess ${ }^{2}$, \\ W. G. Schreiber ${ }^{1}$, and D. Hinderberger ${ }^{2}$ \\ ${ }^{1}$ Section of Medical Physics, Department of Radiology, Mainz University Medical School, Mainz, \\ Germany \\ ${ }^{2}$ Max Planck Institute for Polymer Research, Mainz, Germany
}

Received 22 October 2007; revised 24 January 2008

(C) Springer-Verlag 2008

\begin{abstract}
A mobile and low-cost apparatus for dynamic nuclear polarization (DNP) hyperpolarization based on a Halbach magnet is presented and its performance is demonstrated at room temperature and with three different radical species dissolved in a glycerol-water mixture. DNP enhancement factors for a triarylmethyl-based radical, TEMPOL and a polyelectrolyte spin-labeled with nitroxide radicals are measured as a function of the microwave power, and the extrapolated maximum enhancement factors are compared. The spin-labeled polyelectrolyte shows the best DNP efficiency and ${ }^{1} \mathrm{H}$ DNP enhancement factors up to -50 were observed and a maximum enhancement factor of -80 was projected. The results demonstrate that with the new mobile apparatus reliable DNP experiments can be performed with reasonably high ${ }^{1} \mathrm{H}-\mathrm{DNP}$ enhancements.
\end{abstract}

\section{Introduction}

The application of ${ }^{13} \mathrm{C}$ (or other low- $\gamma$ nuclei) nuclear magnetic resonance (NMR) spectroscopy and imaging for clinical diagnosis has been constrained by the extremely long acquisition times that are required to obtain sufficient signal-to-noise ratios under physiological conditions. Reasons for that are low natural abundance of ${ }^{13} \mathrm{C}$, low concentration of ${ }^{13} \mathrm{C}$-compounds, and physiological temperature, etc. However, this obstacle could be overcome by in vitro hyperpolarization of a ${ }^{13} \mathrm{C}$ containing molecule with long spin-lattice relaxation time via dynamic nuclear polarization (DNP) or parahydrogen-induced polarization and subsequent injection into the animal or patient under study as has been demonstrated recently [1-7]. MR angiography and perfusion measurements can benefit significantly from using hyperpolarized ${ }^{13} \mathrm{C}$-containing molecules since there is no background signal from the thermally polarized ${ }^{13} \mathrm{C}$ nuclei in the tissue, and therefore the contrastto-noise ratio (CNR) approaches the signal-to-noise ratio (SNR) [2-4]. Another important impact of utilizing hyperpolarized fluids for perfusion measurements is that the quantification of a blood flow through the organ of interest (in millime- 
ter per minute per gram tissue) is simplified as compared with the usage of gadolinium-based contrast agents: for hyperpolarized compounds the signal increase because of inflowing contrast agents depends linearly on the contrast agent concentration, which is not the case for Gd-based contrast agents at high concentrations. Of course, reductions of the hyperpolarized signal due to radio frequency (rf) pulses and $T_{1}$ relaxation have to be taken into account.

Another emerging field in medical applications for the use of hyperpolarized substances is molecular or real-time metabolic imaging, e.g., to improve the diagnostic assessment of tumors or to monitor their therapy. Pathologic tissue can be differentiated from normal tissue on the basis of different metabolic pathways within the citrate cycle. As a consequence, the NMR signal intensity of lactate after injection of hyperpolarized ${ }^{13} \mathrm{C}$-pyruvate is significantly elevated in cancer when compared with that in normal tissue [5-7].

However, one major issue of the usage of hyperpolarized substances in medical imaging is the limited lifetime of the hyperpolarized state, thereby restricting in vivo application and detection of the hyperpolarized molecules to roughly three times $T_{1}$. This problem is even more pronounced if the hyperpolarization process cannot take place in the vicinity of the used MR scanner due to safety restrictions or space limitation like in medical MR facilities. In this communication, we report the development of a mobile DNP polarizer based on an intermediatefield Halbach magnet [8].

The use of a mobile permanent magnet design enables the minimization of the transport time from the hyperpolarization site to the object of interest (e.g., animal) resulting in an efficient use of the nonequilibrium magnetization. After the measurement the polarizer can be removed from the MR scanner and the MR imaging (MRI) system is available for routine scanning. Moreover, the Overhauser effect at intermediate field strength is more efficient than at high field. In addition, the penetration depth of microwaves at the corresponding electron paramagnetic resonance (EPR) frequencies $(2-9 \mathrm{GHz})$ is larger, which permits the irradiation of large sample volumes [9, 10].

\section{Materials and Methods}

The Halbach magnet used in this work consists of a total of 576 individual permanent magnets (sintered copper-samarium alloy) that are arranged in Halbach design to form three nested magnet rings. The individual bar magnets are fixed between two aluminum discs which are milled with precise pockets at defined angles [11]. The three magnet rings can be rotated with respect to each other with stepper motors, thus enabling an accurate adjustment of the desired field strength (0.03$0.30 \mathrm{~T}$ ), while the direction of the magnet field is determined by the center magnet ring $[11,12]$. The diameter of the inner bore of the magnet and its height are 81 and $137 \mathrm{~mm}$, respectively. Further details regarding the sweeping of the field and general characteristics of the three-ring magnet are outside of the scope of this paper and will be published elsewhere. From EPR test measurements the homogeneity of the magnetic field at the center of the magnet was estimated to be $100 \mathrm{kHz}(0.1 \mathrm{ml}$ 
sample volume). Including stepper motors and control electronics, the magnet weighs $90 \mathrm{~kg}$. For simple transportation it has been mounted in combination with the microwave source on a table-sized, two-level carriage (Fig. 1).

For the DNP experiments the field strength of the magnet system was adjusted to $0.30 \mathrm{~T}$, corresponding to electron and ${ }^{1} \mathrm{H}$ Larmor frequencies of 8.41 $\mathrm{GHz}$ and $12.77 \mathrm{MHz}$, respectively. For EPR irradiation and NMR detection a Bruker (Karlsruhe, Germany) probehead (MD4EN) originally designed for electron-nuclear double resonance (ENDOR) was used. It consists of a dielectric EPR resonator with NMR coils for irradiation and detection in the rf range (usually used from 1 to approximately $30 \mathrm{MHz}$ ). The electron spin Larmor frequency at a resonance field of $0.30 \mathrm{~T}$ is at the lower limit of the probehead frequency range, and to shift the probehead resonance to this frequency we used a hollow shaft made of ceramics that was attached to the end of the EPR sample tube. Ceramics is a dielectric medium known to shift microwave resonances to lower values without inducing a significant loss in quality factor. A Hewlett Packard HP 8350 B microwave source capable of a maximum output power of $30 \mathrm{~mW}$ and with a tuning range of $1-30 \mathrm{GHz}$ was used. The source output was amplified by a Varian traveling wave tube (TWT) amplifier with a maximum continuous-wave (CW) output power of $20 \mathrm{~W}$ at X-band frequencies $(8-12.4 \mathrm{GHz})$. For NMR detection an APOLLO console (TECMAG, Houston, TX, USA) was employed (10-300 $\mathrm{MHz}$ ), and the duration of the $90^{\circ}$ pulse of the NMR coil for the ENDOR probehead was found to be $12 \mathrm{~ms}$.

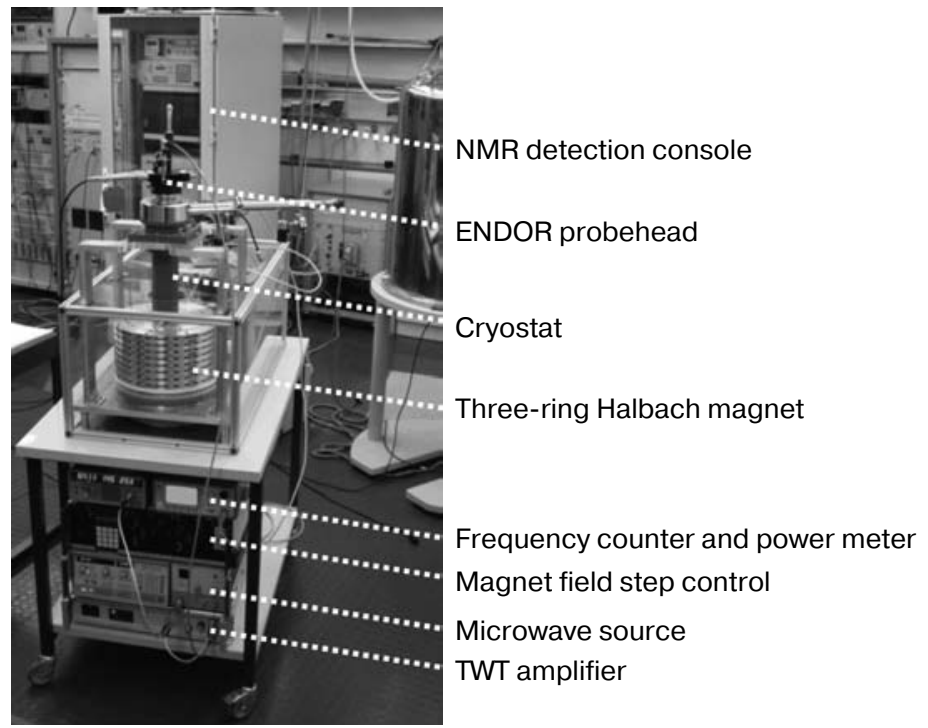

Fig. 1. Mobile DNP polarizer. The complete polarizer is mounted on one rack - the tunable Halbach magnet with cryostat and probehead can be seen on the upper tray, the microwave source and amplifier and the control electronics for the stepper motors are placed on the lower tray. The NMR detection console is visible in the background. 
Three different radicals were used for the DNP experiments: TAM (tris $\{8-$ carboxyl-2,2,6,6-tetra[2-(1-hydroxyethyl)]-benzo(1,2-d:4,5-d' )bis(1,3)dithiole-4yl methyl sodium salt, a kind gift from Nycomed Innovations AB), TEMPOL (4-hydroxy-2,2,6,6-tetramethyl-piperidine-1-oxyl, Sigma, Germany) and a spin-labeled cationic polyelectrolyte poly(DADMAC) (a copolymer of poly(diallyldimethylammonium chloride) with $4 \%$ nitroxide radical-bearing monomers), which has an average molecular weight of $M_{\mathrm{w}}=490000$ (measured by gel permeation chromatography) and a polydispersity of about 3 (a gift from the group of Prof. Andre Laschewsky, Fraunhofer Institut für Angewandte Polymerforschung, Golm, see ref. 13 for a similar synthesis).

Each of the radicals was dissolved in a 55:45 (vol\%) glycerol-water (deionized) mixture to give a radical concentration of $2 \mathrm{mM}$ (TAM concentration, 15 $\mathrm{mM}$ ) and for DNP experiments approximately $0.1 \mathrm{ml}$ of each mixture was transferred into a quartz sample tube with an outer diameter of $3 \mathrm{~mm}$. This solvent

$\mathbf{a}$

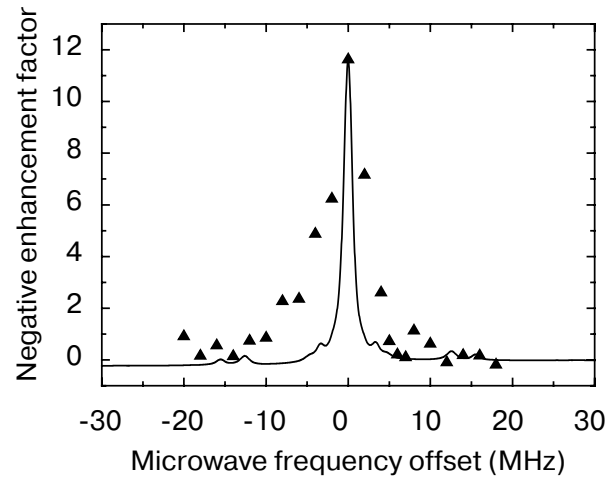

b

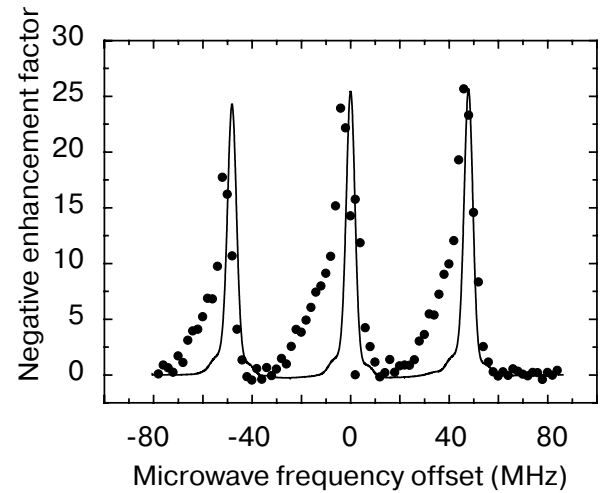

Fig. 2. Comparison of CW EPR spectra with ${ }^{1} \mathrm{H}$ DNP enhancement curves at room temperature. Solid lines depict the EPR spectra, and triangles (circles) the DNP enhancement factors measured for TAM (TEMPOL) as a function of the microwave offset frequency. a TAM at an incident microwave power of $12 \mathrm{~W}$. b TEMPOL radical at an incident microwave power of $6 \mathrm{~W}$. The enhancement curves reflect the measured EPR spectra. For further details see the text. For the poly(DADMAC) radical the CW

EPR spectrum resembles that of the freely movable TEMPOL radical and is not presented here. 
mixture was chosen in order to simulate complex solutions of polarizing agents, polarizable molecules and solvents, which shall finally be used for imaging experiments.

X-band (resonance frequency, about $9.42 \mathrm{GHz}$; magnetic field, about 0.335 T) CW EPR spectra of the three solutions at room temperature were recorded on a MiniScope MS200 benchtop CW EPR spectrometer (Magnettech, Berlin, Germany) with a variable-temperature unit. These CW EPR spectra were used in Fig. 2 for comparison with the DNP enhancement effects. This is a viable approach since the field-dependent changes (e.g., $g$-values) when comparing effects measured at $0.300 \mathrm{~T}$ with spectra measured at $0.335 \mathrm{~T}$ (in particular for TAM) are small and the hyperfine interaction dominating the nitroxide CW EPR spectra at these low field values has no magnetic field dependence.

The NMR spectra (usually 1 scan) were recorded using a Hahn echo sequence (echo time, $0.2 \mathrm{~ms}$ ) and Fourier-transforming half of the measured echo. This allows for refocusing and detecting solely the nuclear magnetization of interest and reduces unwanted background signals from the probehead that are observable when direct free induction decay (FID) detection was employed.

We used $\mathrm{CW}$ irradiation to perform the DNP experiments in our setup. The DNP performance of our device was measured by recording ${ }^{1} \mathrm{H}$ spectra as the used NMR spectrometer was not capable of measuring ${ }^{13} \mathrm{C}$ frequencies at the employed magnetic field $(3.2 \mathrm{MHz}$ at $0.3 \mathrm{~T})$.

The measured ${ }^{1} \mathrm{H}$ spectra showed a line width (full-width at half-maximum) of approximately $6000 \mathrm{~Hz}$ owing mainly to the poor homogeneity of the Halbach magnet. A distinction of peaks stemming from different ${ }^{1} \mathrm{H}$ nuclear spins of glycerol and water was therefore not possible, all lines always contributed to the recorded broad single line spectrum. The DNP enhancement was calculated by dividing the NMR signal without incident microwave irradiation with the NMR signal upon simultaneous microwave irradiation.

\section{Results}

Figure 2 shows the CW EPR spectra at room temperature that were numerically integrated to permit better comparison with the overlaid ${ }^{1} \mathrm{H}$ DNP enhancement curve.

Under the conditions used in this study (field strength, $0.30 \mathrm{~T}$; room temperature) the dipolar electron-nuclear interactions are the dominant DNP mechanisms and therefore the resulting DNP enhancements are negative [8]. For better visualization only absolute values are shown in Fig. 2. For the TAM radical the CW EPR spectrum consists of a narrow central line with a width (peak to peak) of approximately $0.03 \mathrm{mT}$ and an array of ${ }^{13} \mathrm{C}$ satellite lines, which are reflected in the ${ }^{1} \mathrm{H}$ DNP enhancement curve. The line width of the enhancement curve is increased in comparison with the CW EPR spectrum due to the saturation of the EPR spectrum during the DNP experiments, and due to a strong overcoupling of the used microwave resonator. 
The EPR spectrum of the TEMPOL radical shows a line width significantly larger than that of the TAM radical (center line width, 0.13 and $0.03 \mathrm{mT}$ for TEMPOL and TAM, respectively). This is a result which can be expected for nitroxide-based radicals because of unresolved ${ }^{1} \mathrm{H}$ hyperfine splittings [14]. For TEMPOL, the ${ }^{1} \mathrm{H}$ DNP enhancement curve closely follows the EPR spectrum with the exception of the left flanks of the individual EPR lines. Since we do not observe such huge flanks when performing DNP experiments with the ENDOR probehead in an electromagnet at $0.34 \mathrm{~T}$ with much lower incident microwave power, this may also be an effect of EPR spectral saturation. Additionally, it can be seen from Fig. $2 b$ that the DNP enhancement is strongest at EPR irradiation of the high-frequency line $\left[\Delta m_{I}\left({ }^{14} \mathrm{~N}\right)=-1\right]$ of the TEMPOL EPR spectrum. Theoretically, if all three lines were equal in amplitude, and if the saturation of one resonance would not affect the other resonances, it would be expected that all three lines demonstrate similar DNP enhancement during irradiation of any of the three lines. The differences observed in Fig. 2b may occur due to frequency steps chosen too large when sweeping the incident microwave frequency, which may result in missing the true maxima of the nitroxide EPR peaks.

In the next step we examined which radical gives the best DNP performance in the proposed mobile DNP polarizer. Therefore, the dependence of DNP enhancement on microwave power for the different radicals was measured at room temperature and the results are depicted in Fig. 3.
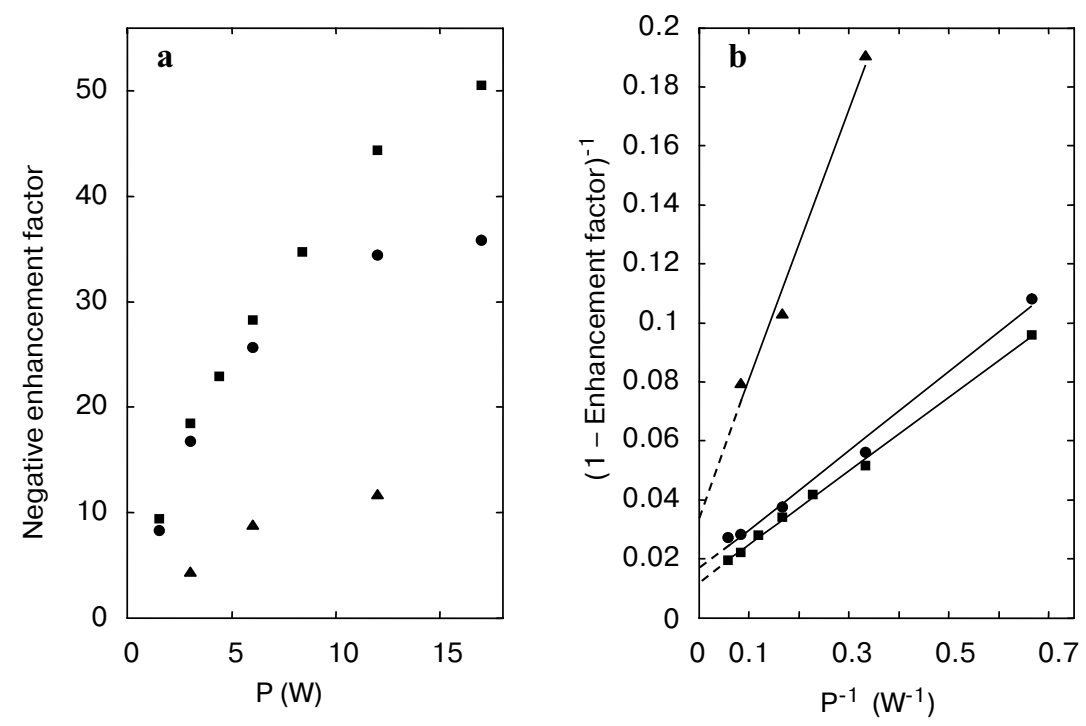

Fig. 3. a Dependence of DNP enhancement as a function of the microwave power for the three radi-

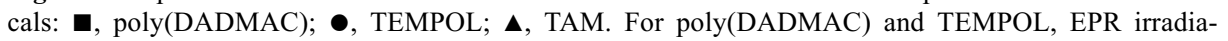
tion was performed at the lowest spectral line. The DNP enhancement increases with increasing microwave power for all radicals. b Determination of the maximum enhancement factor. Solid lines represent linear regression to the data points of each radical. The extrapolation to $1 / P>0$ indicates the theoretically achievable maximum enhancement factor for each radical. 
From Fig. 3a it becomes obvious that for all radicals the measured DNP enhancement increases with increasing microwave power, which results in an improved saturation of the EPR spectrum. At small microwave powers, TEMPOL and poly(DADMAC) show almost equal DNP enhancements, whereas at high microwave powers the DNP enhancement of poly(DADMAC) significantly exceeds that of TEMPOL. This can be rationalized from the saturation behavior of the corresponding CW EPR spectra, since the narrower lines of TEMPOL (as compared with the poly(DADMAC) line widths) are more easily saturated. The TAM radical gives the smallest DNP enhancement and the weakest dependence on microwave power in our experimental setup. The highest observed negative DNP enhancements for poly(DADMAC), TEMPOL and TAM were 50 (at $17 \mathrm{~W}$ microwave power), 36 (at $17 \mathrm{~W}$ microwave power) and 12 (at $12 \mathrm{~W}$ microwave power), respectively.

The maximum DNP enhancement that can be achieved with our experimental conditions can be obtained by plotting the reciprocal Overhauser enhancement $(1-\text { enhancement factor })^{-1}$ against the reciprocal EPR power level $P^{-1}$ which should show a linear dependence $[10,14,15]$. The data points in Fig. $3 \mathrm{~b}$ demonstrate the expected linear relation. Applying a linear fit to these data points and extrapolating it to zero yields $(1-\text { maximum enhancement factor })^{-1}$ and the maximum enhancement factor can be extracted that would occur at complete saturation of the EPR transition. The maximum enhancement factors obtained by this method are -80 for poly(DADMAC), -60 for TEMPOL and -27 for the TAM radical.

\section{Discussion}

Our results demonstrate that it is possible to perform reliable DNP experiments with a new mobile apparatus, and that reasonably high ${ }^{1} \mathrm{H}-\mathrm{DNP}$ enhancements can be achieved.

Despite the fact that our measured maximum enhancements of $-50,-36$ and -12 are slightly lower than those reported for similar systems and hardware setups $[14,17]$, it is noteworthy that the ENDOR probehead employed in this study is used at EPR resonance conditions $(8.41 \mathrm{GHz} / 0.30 \mathrm{~T})$ for which this commercial probehead is not optimized. The microwave absorption characteristics are rather poor: it is highly overcoupled and microwave reflections already significantly diminish the performance. Theoretically, DNP enhancements at $0.30 \mathrm{~T}$ should even be slightly better than those at $0.33 \mathrm{~T}$, which would be the standard magnetic field and frequency range for EPR. It is likely that we could not make use of the possible additional enhancement due to the rather poor EPR probehead characteristics at $0.3 \mathrm{~T}$. We are therefore currently building a doubleresonance probehead that is optimized for EPR performance at $8.4 \mathrm{GHz}$, and we expect a significantly improved EPR and DNP performance (e.g., less microwave power to achieve similar NMR signal enhancements).

Furthermore, there is also a discrepancy between the maximum achievable enhancement factor for the different radicals that can be obtained in our device 
and the ones that are predicted by theory. For the TAM radical dissolved in water, a maximum DNP enhancement at $0.3 \mathrm{~T}$ and $26{ }^{\circ} \mathrm{C}$ of 92 (for $10 \mathrm{mM}$ solutions of TAM) was proposed by Wind and Ardenkjaer-Larsen [10]. We obtain a significantly smaller DNP enhancement for TAM in the water-glycerol mixture. The theoretically predicted maximum ${ }^{1} \mathrm{H}-\mathrm{DNP}$ enhancement factor in systems containing ${ }^{14} \mathrm{~N}$-nitroxide radicals at room temperature is -110 [15]. This value is significantly greater than the ones that can be obtained in the mobile polarizer for our ${ }^{14} \mathrm{~N}$-nitroxide radicals dissolved in a 55:45 mixture of glycerol and water $(-80$ and -60 for poly(DADMAC) and TEMPOL, respectively).

However, our results are qualitatively comparable with results gained on waterglycerol mixtures at very low fields [15]. This lower DNP enhancement can be due to the fact that the dipolar couplings between the unpaired electron spins and the proton nuclear spins of the water molecules are more efficient, as has also been reported previously for TAM and nitroxides [10, 14, 16].

This latter explanation may possibly have two sources of origin: first, the smaller size of the water molecules compared with the glycerol molecules allows the water molecules to closely approach the radicals resulting in more efficient transfer of electron spin polarization to the ${ }^{1} \mathrm{H}$ nuclear spins. In addition, glycerol also possesses five non-hydrogen-bonding protons, which due to the broad overall NMR line width we also detect in our NMR spectra. The polarization transfer to these nuclear spins may well be different from that to nuclear spins located on H-bond forming hydrogens. Second, due to the added glycerol the overall tumbling rate of the radicals in solution is slower than that in pure water, which also affects the polarization transfer efficiency [15]. Surprisingly, this is particularly true for the high-molecular-weight polymer poly(DADMAC), which in our study shows the highest DNP efficiency.

The fact that the use of solutions of a nitroxide spin-labeled poly(DADMAC) at incident microwave powers greater than $12 \mathrm{~W}$ gives up to 1.5 times increased DNP enhancements as compared with free TEMPOL is the most striking result concerning the used polarizing agents. The nitroxide radicals are distributed statistically along the polyelectrolyte chain and on average every 25 th repeat unit holds a nitroxide side group (4\% labeling grade). Due to the statistical nature of the labeling and the manifold of conformations that a polyelectrolyte chain in solution may adopt [18], a broad range of intramolecular and intermolecular radical-radical distances and hence of dipolar couplings between electron spins can be expected to be found. Indeed, from measurements of the dipolar electron-electron interactions by either CW EPR or the double electron-electron resonance method [19] (J. Heller et al., Max Planck Institute for Polymer Research, Mainz, Germany, unpubl.) we could estimate that nitroxide radicals of poly(DADMAC) in such a solvent feature mainly dipolar couplings in the range of approximately 2-40 $\mathrm{MHz}$ (amounting to distances of about 1-5 nm). Due to the dipolar nature of the enhancement, a "fine-tuning" of the prevalent electron-electron dipolar couplings to the Larmor frequency of the nucleus of interest can help optimizing the Overhauser-type DNP enhancement [20, 21]. In this respect, the spin-labeled poly(DADMAC) can be seen as a broadband polarizing agent: it apparently shows better enhancements in ${ }^{1} \mathrm{H}$ NMR spectra and can be estimated to also increase 
the DNP effect as compared to TEMPOL solutions for ${ }^{13} \mathrm{C} \mathrm{NMR}$, one of the nuclei most promising for clinical applications (resonance frequency of about $3.2 \mathrm{MHz}$ at $0.3 \mathrm{~T}$ ).

Since our mobile polarizer is designed and built for final use in a clinical environment and thus for preparing hyperpolarized contrast agents for experiments in animals and eventually humans, the spin-labeled polyelectrolyte has additional advantages. Although poly(DADMAC) itself has been shown to be toxic towards aquatic organisms [22], many natural water-soluble polyelectrolytes could be spinlabeled and used as nontoxic polarizing agents (e.g., polysaccharides or chitosan derivatives). Furthermore, one can devise schemes to extract spin-labeled polyelectrolytes from the solutions before injection by using them as a fixed polyelectrolyte gel matrix (similar to the system in ref. 17) or by extracting them through electrostatic interactions (e.g., by adding oppositely charged polyelectrolytes or surfaces).

We will expand our range of applied polarizing agents and test the concentration dependence of the effective DNP enhancement for various systems in order to identify chemical systems that can potentially be used for testing in animals.

We are also currently investigating the low-temperature DNP properties of our apparatus. In our system, with the employed cryostat and the use of liquid-helium-flow cooling system (Oxford Instruments, Oxford, UK) we can theoretically achieve temperatures down to $4 \mathrm{~K}$. At low temperatures it is expected that due to the increased Boltzmann polarization of the electron spins the achievable DNP enhancement may increase. Also, the mechanism for transfer of polarization from electron spins to nuclear spins changes from an Overhauser-type mechanism to either the solid effect or thermal mixing [23]. We have now started a study concerning the features of our systems at low temperatures in order to achieve higher DNP enhancements in our mobile apparatus.

\section{Acknowledgments}

We thank Joachim Storsberg and Andre Laschewsky, Fraunhofer Institut für Angewandte Polymerforschung, Golm, for the synthesis of the spin-labeled poly(DADMAC). Financial support from the German Research Foundation (grant nr. SCHR 687/2-3) and funding by the research funds of the Johannes Gutenberg University, Mainz, are appreciated.

\section{References}

1. Mansson, S., Johansson, E., Magnusson, P., Chai, C.-M., Hansson, G., Petersson, J.S., Stahlberg, F., Golman, K.: Eur. Radiol. 16, 57-67 (2006)

2. Golman, K., Ardenkjaer-Larsen, J.H., Svensson, J., Axelsson, O., Hansson, G., Hansson, L., Johannesson, H., Leunbach, I., Mansson, S., Petersson, J.S., Petersson, G., Servin, R., Wistrand, L.-G.: Acad. Radiol. 9, 507-510 (2002)

3. Svensson, J., Mansson, S., Johansson, E., Petersson, J.S., Olsson, L.E.: Magn. Reson. Med. 50, 256-262 (2003) 
4. Johansson, E., Olsson, L.E., Mansson, S., Petersson, J.S., Golman, K., Ståhlberg, F., Wirestam, R.: Magn. Reson. Med 52, 1043-1051 (2004)

5. Golman, K., Ardenkjaer-Larsen, J.H., Petersson, J.S., Mansson, S., Leunbach, I.: Proc. Natl. Acad. Sci. USA 100, 10435-10439 (2003)

6. Golman, K., in't Zandt, R., Lerche, M., Pehrson, R., Ardenkjaer-Larsen, J.H.: Cancer Res. 66, 10855-10860 (2006)

7. Battacharya, P., Chekmenev, E.Y., Perman, W.H., Harris, K.C., Lin, A.P., Norton, V.A., Tan, C.T., Ross, B.D., Weitekamp, D.P.: J. Magn. Reson. 186, 150-155 (2007)

8. Halbach, K.: Nucl. Instrum. Methods 169, 1-10 (1980)

9. Abragam, A., Goldman, M.: Rep. Prog. Phys. 41, 395-467 (1978)

10. Wind, R.A., Ardenkjær-Larsen, J.-H.: J. Magn. Reson. 141, 347-354 (1999)

11. Bauer, C., Raich, H.P., Jeschke, G., Soltner, H., Blümler P.: Magnetsystem mit variabler Feldstärke, German Patent Application 102006031 425.5-24 (5.7.2006)

12. Raich, H., Blümler, P.: Concepts Magn. Reson. B: Magn. Reson. Eng. 23B, $16-25$ (2004)

13. Glinel, K., Moussa, A., Jonas, A.M., Laschewsky, A.: Langmuir 18, 1408-1412 (2002)

14. Franklin Benial, A.M., Ichikawa, K., Murugesan, R., Yamada, K., Utsumi, H.: J. Magn. Reson. 182, 273-282 (2006)

15. Nicholson, I., Lurie, D.J., Robb, F.J.L.: J. Magn. Reson. B 104, 250-255 (1994)

16. Ardenkjaer-Larsen, J.H., Laursen, I., Leunbach, I., Ehnholm, G., Wistrand, L.-G., Petersson, J.S., Golman, K.: J. Magn. Reson. 133, 1-12 (1998)

17. McCarney, E.R., Armstrong, B.D., Lingwood, M.D., Han, S.: Proc. Natl. Acad. Sci. USA 104, 1754-1759 (2007)

18. Förster, S., Schmidt, M.: Adv. Polym. Sci. 120, 51 (1995)

19. Berliner, L.J., Eaton, G.R., Eaton, S.S. (eds.): Distance Measurements in Biological Systems by EPR. Biological Magnetic Resonance, vol. 19. Kluwer Academic, New York (2000)

20. Hu, K.-N., Yu, H., Swager, T.M., Griffin, R.G.: J. Am. Chem. Soc. 126, 10844-10845 (2004)

21. Song, C., Hu, K.-N., Joo, C.-G., Swager, T.M., Griffin, R.G.: J. Am Chem. Soc. 128, 11385-11390 (2006)

22. de Rosemond, S.J.C., Liber, K.: Environ. Toxicol. Chem. 23, 2234-2242 (2004)

23. Gerfen, G.J., Becerra, L.R., Hall, D.A. Griffin, R.G., Temkin, R.J., Singel, D.J.: J. Chem. Phys. 102, 9494-9497 (1995)

Authors' address: Dariush Hinderberger, Max Planck Institute for Polymer Research, Ackermannweg 10, 55128 Mainz, Germany

E-mail: hinderberger@mpip-mainz.mpg.de 